Practical Rails Projects

Eldon Alameda 


\section{Practical Rails Projects}

\section{Copyright $\odot 2007$ by Eldon Alameda}

All rights reserved. No part of this work may be reproduced or transmitted in any form or by any means, electronic or mechanical, including photocopying, recording, or by any information storage or retrieval system, without the prior written permission of the copyright owner and the publisher.

ISBN-13 (pbk): 978-1-59059-781-1

ISBN-10 (pbk): 1-59059-781-8

Printed and bound in the United States of America 987654321

Trademarked names may appear in this book. Rather than use a trademark symbol with every occurrence of a trademarked name, we use the names only in an editorial fashion and to the benefit of the trademark owner, with no intention of infringement of the trademark.

Lead Editors: Ben Renow-Clarke and Chris Mills

Technical Reviewer: Paul Bentley

Editorial Board: Steve Anglin, Ewan Buckingham, Tony Campbell, Gary Cornell, Jonathan Gennick, Jason Gilmore, Kevin Goff, Jonathan Hassell, Matthew Moodie, Joseph Ottinger, Jeffrey Pepper, Ben Renow-Clarke, Dominic Shakeshaft, Matt Wade, Tom Welsh

Senior Project Manager: Tracy Brown Collins

Copy Editor: Heather Lang

Assistant Production Director: Kari Brooks-Copony

Senior Production Editor: Laura Cheu

Compositor: Pat Christenson

Proofreader: Lori Bring, Christy Wagner, Elizabeth Berry

Indexer: Becky Hornyak

Artist: April Milne

Cover Designer: Kurt Krames

Manufacturing Director: Tom Debolski

Distributed to the book trade worldwide by Springer-Verlag New York, Inc., 233 Spring Street, 6th Floor, New York, NY 10013. Phone 1-800-SPRINGER, fax 201-348-4505, e-mail orders-ny@springer-sbm. com, or visit http://www. springeronline.com.

For information on translations, please contact Apress directly at 2855 Telegraph Avenue, Suite 600, Berkeley, CA 94705. Phone 510-549-5930, fax 510-549-5939, e-mail info@apress.com, or visit http:// www. apress.com.

The information in this book is distributed on an "as is" basis, without warranty. Although every precaution has been taken in the preparation of this work, neither the author(s) nor Apress shall have any liability to any person or entity with respect to any loss or damage caused or alleged to be caused directly or indirectly by the information contained in this work.

The source code for this book is available to readers at http://Www. apress.com. 


\section{Contents at a Glance}

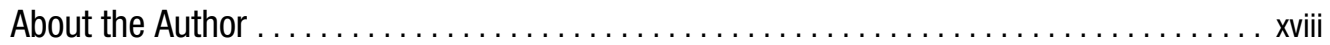

About the Technical Reviewer...................................... xix

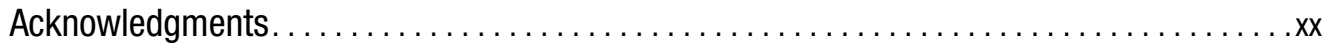

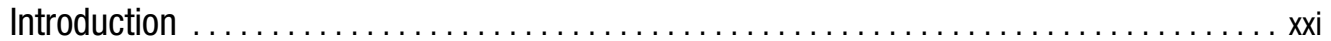

\section{PART 1 Making the Right Preparations (Don't Skip This Part)}

CHAPTER 1 Building a Development Environment $\ldots \ldots \ldots \ldots \ldots \ldots \ldots$

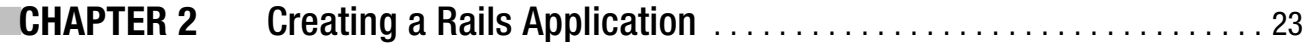

\section{PART 2 Monkey Tasks: Managing a Daily Task List}

CHAPTER 3 Implementing a User Registration and Authentication System . . 35

CHAPTER 4 Building a Daily To-Do Manager...................... 57

CHAPTER 5 Enhancing Monkey Tasks $\ldots \ldots \ldots \ldots \ldots$

\section{PART 3 Exercisr}

CHAPTER 6 Developing a REST-Based Application ................... 93

CHAPTER 7 Adding Graphs to Our Application .......................... 155

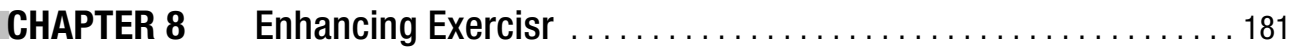

PART 4 Simple Blogs

CHAPTER 9 Building a Blog Using Typo ...................... 187

CHAPTER 10 Building a Simple Blog Engine $\ldots \ldots \ldots \ldots \ldots \ldots \ldots \ldots \ldots \ldots \ldots$

CHAPTER 11 Enhancing Our Blogs ............................. 265 


\section{PART 5 Building a Web Comic Using Caching}

CHAPTER 12 Building Our Base System with Page Caching............. 271

CHAPTER 13 Implementing Advanced Caching ...................... 299

CHAPTER 14 Enhancing the Web Comic .......................... 315

\section{PART 6 Church Community Site}

CHAPTER 15 Managing Users and Profiles $\ldots \ldots \ldots \ldots \ldots \ldots \ldots \ldots \ldots \ldots \ldots \ldots \ldots$

CHAPTER 16 Rounding out the Community ...................... 365

CHAPTER 17 Enhancing the Church Community Application ............. 389

\section{PART 7 GamingTrend}

CHAPTER 18 Understanding the Problems of the Legacy PHP Site . . . . . . . 397

CHAPTER 19 Converting Game Records ....................... 419

CHAPTER 20 Supporting News ................................... 501

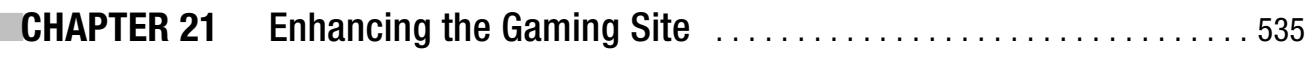

\section{PART 8 Integrating with a RESTful Application Using Edge Rails (Rails 2.0)}

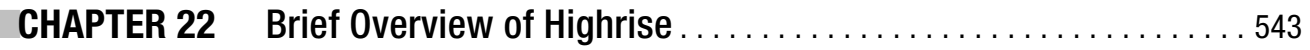

CHAPTER 23 Integrating to the Highrise REST API.................... 549

CHAPTER 24 Building the Appointment Scheduler ...................... 559

CHAPTER 25 Enhancing Our Rails 2.0 Application.................. 585 


\section{Contents}

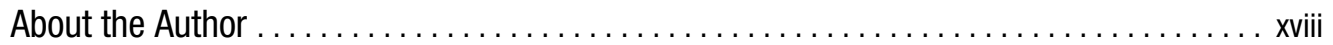

About the Technical Reviewer................................... xix

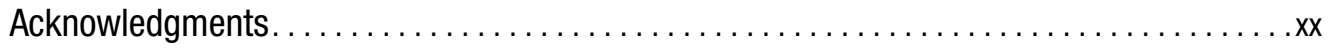

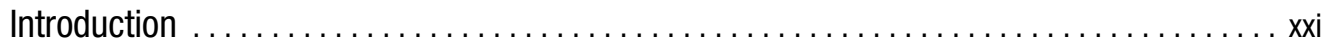

\section{PART 1 Making the Right Preparations (Don't Skip This Part)}

CHAPTER 1 Building a Development Environment ................ 3

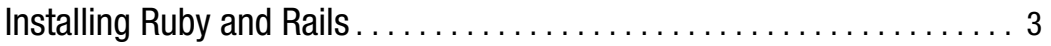

Installing on Linux............................. 4

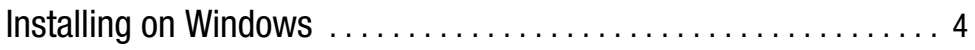

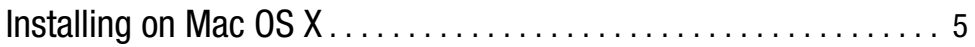

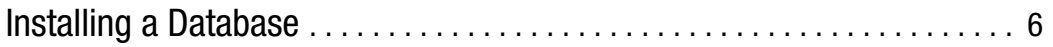

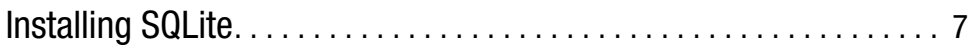

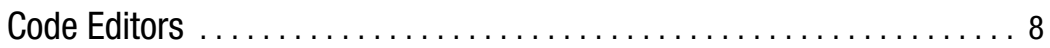

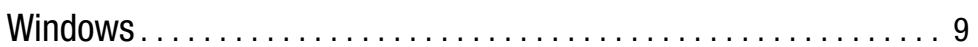

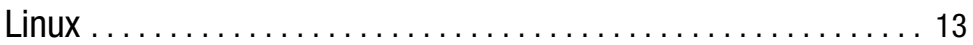

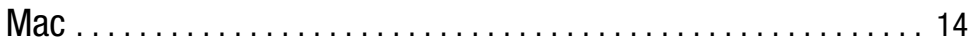

Text Editor Recommendations . ....................... 15

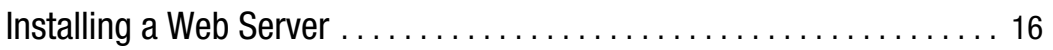

WEBBrick .................................... 16

Mongrel. .................................... 17

Extra Tips that You'll Want to Adopt (Eventually).............. 18

Use a Version Control System ........................ 19

Automating Deployment with Capistrano $\ldots \ldots \ldots \ldots \ldots \ldots \ldots 20$

Summary ......................................... 22 
CHAPTER 2 Creating a Rails Application $\ldots \ldots \ldots \ldots \ldots \ldots \ldots \ldots \ldots$

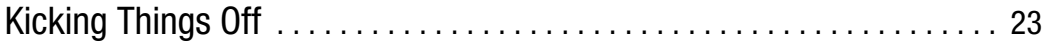

Step 1: Create the Project......................... 23

Step 2: Configure Database Settings. . . . . . . . . . . . . . 27

Step 3: Test the Application......................... 28

Step 4: Install Style Sheets......................... 29

Step 5: Freeze Rails........................... 30

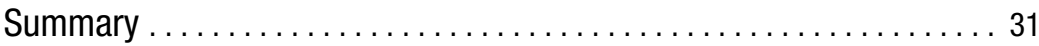

\section{PART 2 Monkey Tasks: Managing a Daily Task List}

CHAPTER 3 Implementing a User Registration and Authentication System ........................................ 35

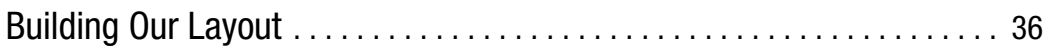

Our Initial Layout .............................. 37

Our First View Template........................... 39

Adding User Registration and Authentication ................. 40

Configuring Acts as Authenticated .................... 43

Building User Registration . . . . . . . . . . . . . . . . . . . . 46

Sending E-mail Notifications $\ldots \ldots \ldots \ldots \ldots \ldots \ldots \ldots \ldots$

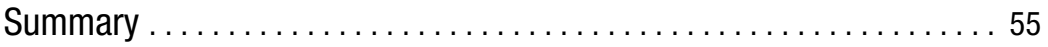

CHAPTER 4 Building a Daily To-Do Manager ................. 57

Creating Our First Model: task $\ldots \ldots \ldots \ldots \ldots \ldots \ldots \ldots \ldots \ldots$

Creating the Task Controller ........................... 60

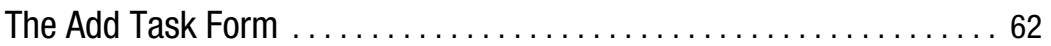

A Better Date Selector ..............................6 63

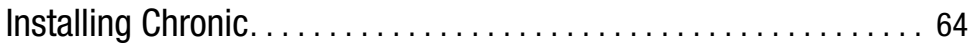

The schedule Model. ............................... 66

The todo Model .................................... 67

Making Our Task Lists Work. ......................... 69

Making Our Daily Schedule Work $\ldots \ldots \ldots \ldots \ldots \ldots \ldots \ldots \ldots \ldots \ldots$

Moving Tasks to the Schedule ....................... 72

Displaying Our Schedule ......................... 74 


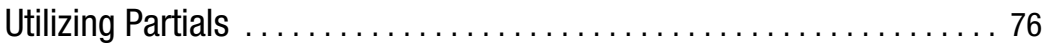

Marking Tasks Complete .......................... 78

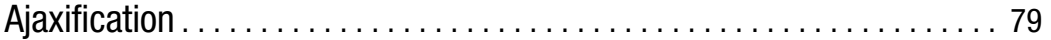

Sortable Elements. ............................ 79

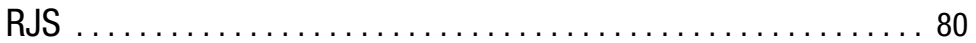

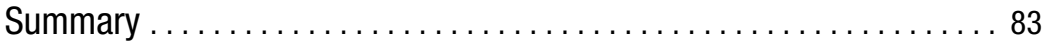

CHAPTER 5 Enhancing Monkey Tasks $\ldots \ldots \ldots \ldots \ldots \ldots$

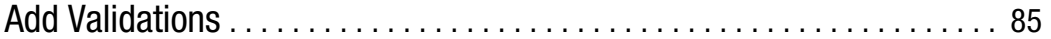

Edit a Task ...................................... 85

Add a Calendar ................................... 85

Navigate Previous Days........................... 86

Capture the Estimated Time for Each Task ................. 86

Display Percentage Completed $\ldots \ldots \ldots \ldots \ldots \ldots \ldots \ldots \ldots \ldots$

Develop an iPhone Interface $\ldots \ldots \ldots \ldots \ldots \ldots \ldots \ldots \ldots \ldots \ldots$

Optimize Database Queries........................... 87

Move Code into Models . ........................... 87

Freeze the Chronic Gem . . . . . . . . . . . . . . . . . . . . . . . 88

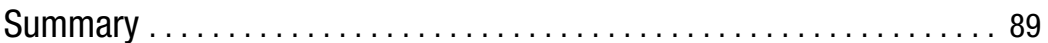

\section{PART 3}

CHAPTER 6 Developing a REST-Based Application ............... 93

REST-Based Development $\ldots \ldots \ldots \ldots \ldots \ldots \ldots \ldots \ldots \ldots \ldots$

So What Is REST? ............................. 94

The Value of REST .............................. 95

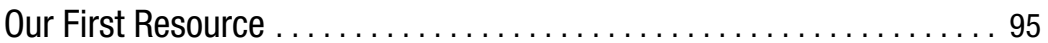

RESTful Tools................................ 95

Building the Exercise Resource ...................... 100

Adding RESTful Authentication........................... 104

Refining the Look ................................... 111

Creating a New User ................................ 113

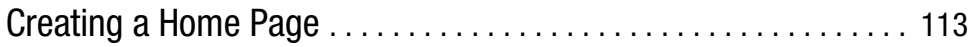

Completing the Exercise Resource ....................... 115

Building the Model Associations....................... 116

Rescoping the Exercise Controller ................... 116

The Exercise Views ............................. 119 
The Workout Resource. ............................ 121

The Workout Model and Associations . . . . . . . . . . . . . . . . 122

The Workout Controller ............................. 123

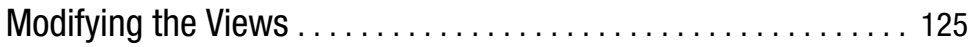

Capturing Our Workouts ............................ 127

Building Our Activities Model and Associations . . . . . . . . . . . 128

Modifying the Activities Routes. ..................... 130

Modifying the Activities Controller .................... 131

Modifying Activities View Templates ..................... 134

Modifying the Show Method for a Workout .............. 136

Improving the Add Activity Form . . . . . . . . . . . . . . . 137

Tracking Fitness Goals. . . . . . . . . . . . . . . . . . . . . . . . . 139

Modifying Our Models ........................... 140

Setting Up a Nested Route............................ 140

Configuring Our Controllers ........................... 141

Configuring Our Views ............................ 145

Capturing the Last Result .......................... 149

Exploring the RESTful Interface . . . . . . . . . . . . . . . . . 149

Summary ....................................... 153

CHAPTER 7 Adding Graphs to Our Application .................. 155

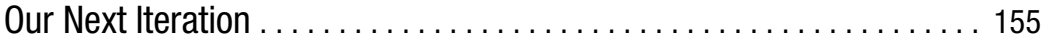

Graphing Options in Rails ........................... 155

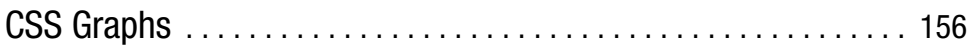

Sparklines.................................... 159

Gruff Graphs................................. 163

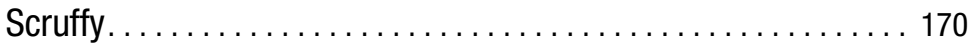

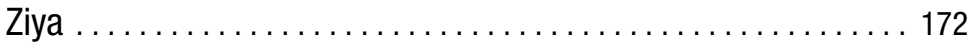

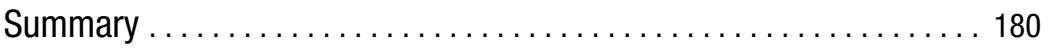

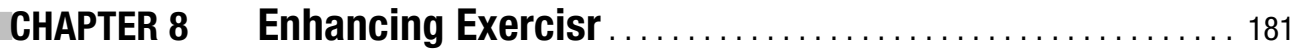

Add RJS to the Interface .............................. 181

Create a Calendar Showing When You Worked Out .............. 182

Cache Reports................................ 182

Make the Home Page RESTful ......................... 182

Develop More Graphs............................. 182

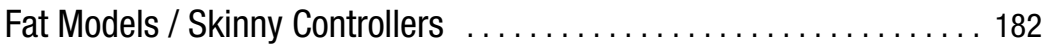

Develop Social Networking Features..................... 183

Summary ....................................... 183 


\section{PART 4 Simple Blogs}

CHAPTER 9 Building a Blog Using Typo 187

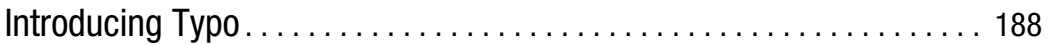

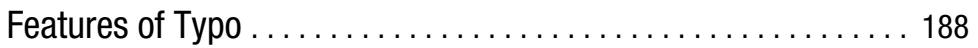

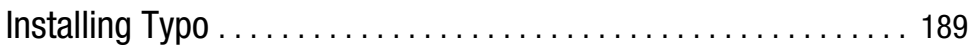

Activating Our Typo Blog .......................... 190

The Rails Startup Process .............................. 192

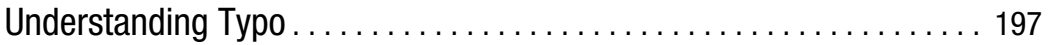

Understanding the Database . . . . . . . . . . . . . . . . . . . 197

How Rails Routes Requests . . . . . . . . . . . . . . . . . . . 201

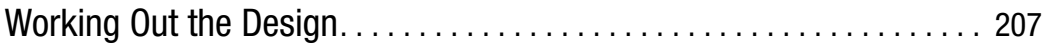

Using Typo ..................................... 209

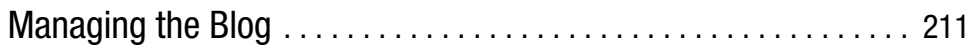

Add the Content. ............................. 212

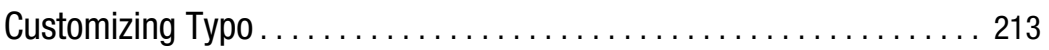

Managing Themes .............................. 213

Exploring a Theme ................................ 214

Building a Custom Theme ......................... 214

Creating a Sidebar Component....................... 220

Customizing a Sidebar Component .................... 221

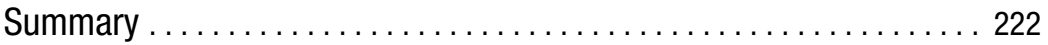

CHAPTER 10 Building a Simple Blog Engine $\ldots \ldots \ldots \ldots \ldots \ldots \ldots \ldots$

So What Are We Going to Build? ..................... 223

Building Our First Models . . . . . . . . . . . . . . . . . . . . . . . 224

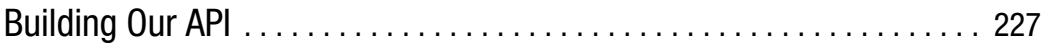

The MetaWeblog Service API . . . . . . . . . . . . . . . . 230

Adding Blogger Support......................... 233

Supporting Images .............................. 246

Building the Public-Facing Side of Our Blog . . . . . . . . . . . 253

Creating a Basic Layout .......................... 253

Editing the Application Controller.................... 256

Creating the Home Page $\ldots \ldots \ldots \ldots \ldots \ldots \ldots \ldots \ldots \ldots \ldots \ldots$

Viewing a Single Post ........................... 260

Adding a Category Filter. . . . . . . . . . . . . . . . . . . 262

Building an RSS Feed ............................ 263

Summary ...................................... 264 


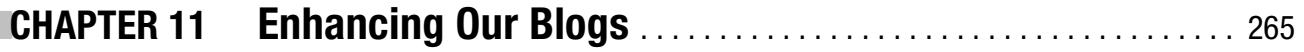

Develop the Blogger API ............................ 265

Build a Blog Using Mephisto .......................... 265

Customize Typo................................... 265

Move Authentication Out of Methods .................... 266

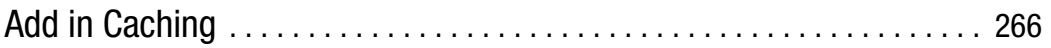

Add Comments and Akismet Spam Filtering . . . . . . . . . . . 266

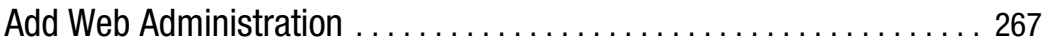

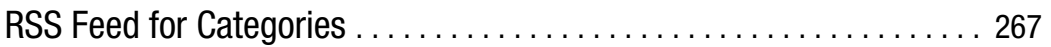

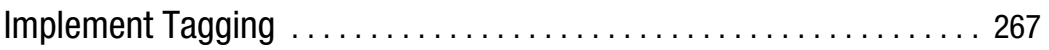

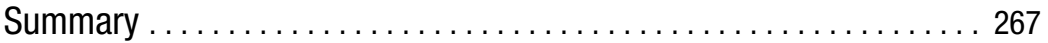

\section{PART 5 Building a Web Comic Using Caching}

CHAPTER 12 Building Our Base System with Page Caching ........ 271

A Basic Administration System . . . . . . . . . . . . . . . . . . . 273

Uploading Comics .............................. 273

Our Comic Model. .................................... 275

Modifying Our Routes.......................... 278

Modifying the Scaffolding ........................ 278

Creating a Comic ............................... 282

A Simple Authentication System. . . . . . . . . . . . . . . . 284

Limiting Access ............................ 286

The Public-Facing Side . . . . . . . . . . . . . . . . . . . . . . . . 287

Page Caching ................................... 289

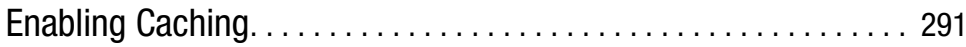

Cleaning Up the Cache . . . . . . . . . . . . . . . . . . . . . . . 294

Summary ....................................... 297

CHAPTER 13 Implementing Advanced Caching ................... 299

The Members Controller .......................... 300

Limiting Access to Subscribers. ........................ 301

Caching Our Members Pages ......................... 304

Expiring Action Caching ............................ 305 


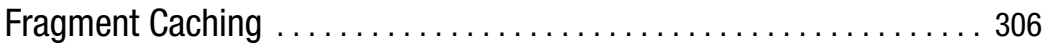

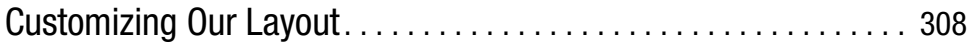

Clearing Our Fragment Cache....................... 313

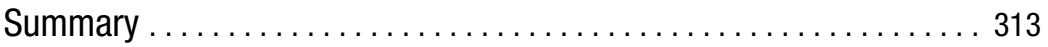

CHAPTER 14 Enhancing the Web Comic ...................... 315

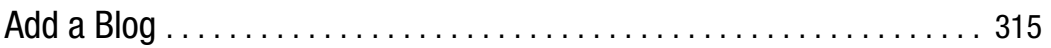

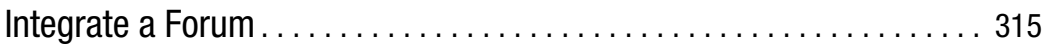

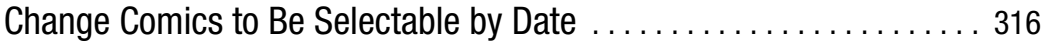

Enhance the Authentication System ...................... 316

Summary ......................................... 317

\section{PART 6 Church Community Site}

CHAPTER 15 Managing Users and Profiles $\ldots \ldots \ldots \ldots \ldots \ldots \ldots \ldots \ldots$

Installing Restful Authentication. ....................... 321

So What Are We Going to Build? ........................ 324

Creating Our Shared Layout ........................ 325

The Avatar Model . . ................................. 327

The User Details Model . . . . . . . . . . . . . . . . . . . . . 329

Creating a Sample User .......................... 331

The Profile Controller .............................. 333

Editing User Details and Avatars ....................... 336

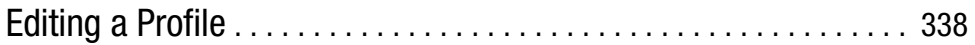

Viewing a Profile ............................... 340

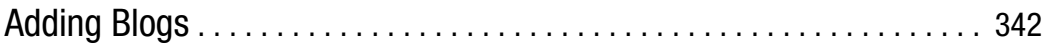

Post Controller Methods and Templates ................. 344

Adding Blog Summaries to Our User Page . . . . . . . . . . . . . . . . . 350

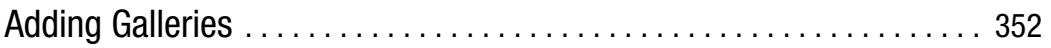

Adding Photos .................................... 355

Galleries and Photo Controllers ........................... 357

Creating a New Gallery . . . . . . . . . . . . . . . . . . . . . 358

Viewing a Specific Gallery . . . . . . . . . . . . . . . . . . . 359

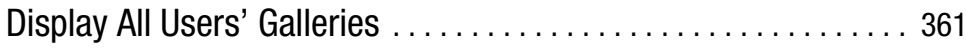

Summary ......................................... 363 
Building the Community Home Page $\ldots \ldots \ldots \ldots \ldots \ldots \ldots \ldots . \ldots . \ldots . \ldots$

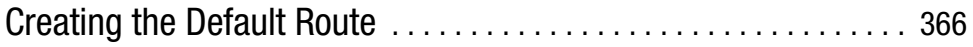

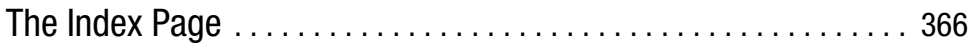

Building the Home Page ........................ 371

Adding a Directory of Users $\ldots \ldots \ldots \ldots \ldots \ldots \ldots \ldots \ldots \ldots \ldots \ldots \ldots$

Generating an Alphabetical Index.................... 373

Creating a Route ................................. 373

Adding the Directory Method $\ldots \ldots \ldots \ldots \ldots \ldots \ldots \ldots \ldots \ldots \ldots \ldots$

Editing Our View Template . ........................... 374

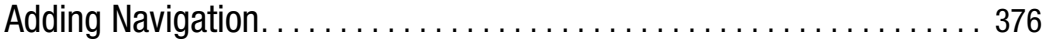

The Upper Navigation . . . . . . . . . . . . . . . . . . . . . 376

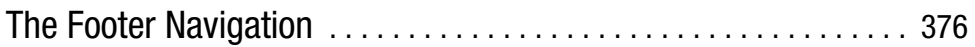

Adding Comments .................................... 377

Installing acts_as_commentable ..................... 377

Displaying Comments............................ 379

Adding Comments. ................................. 382

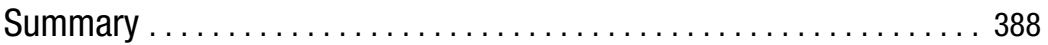

CHAPTER 17 Enhancing the Church Community Application........ 389

Create a User Import or Sign-Up Process. . . . . . . . . . . . . . . . . . 389

Batch Upload Photos Using SWFupload . . . . . . . . . . . . . . . . 390

Add an RSS Feed for Each User . . . . . . . . . . . . . . . . . . . . 390

Add Friends List Functionality . . . . . . . . . . . . . . . . . . . . . . . 391

Enhance the Home Page ................................ 391

Clean Up Some of Our Ruby Code ...................... 391

Move Code into Partials. ............................. 392

Implement Kropper for User Profile Images . . . . . . . . . . . . . . . . . 393

Add Caching ........................................ 394

Summary ...................................... 394

\section{PART 7 GamingTrend}

CHAPTER 18 Understanding the Problems of the Legacy PHP Site . . 397

A Quick Tour of the Current System . . . . . . . . . . . . . . . . 398

A Look at the Existing Code...................... 400

Issues with the Old System ........................ 402 
Setting Up Our Application $\ldots \ldots \ldots \ldots \ldots \ldots \ldots \ldots \ldots \ldots \ldots \ldots 402$

Utilizing the Console $\ldots \ldots \ldots \ldots \ldots \ldots \ldots \ldots \ldots \ldots \ldots, \ldots \ldots \ldots$

Utilizing Ext-JS to Create a Better Interface. .................. 405

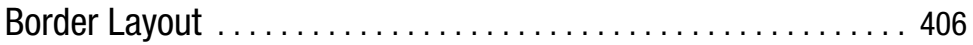

Grid ..................................... 407

Dialogs .................................... 409

Message Box .............................. 409

Installing Ext-JS into Our Rails Application . ................ 410

Automating Ext-JS Installation .......................... 411

Creating the Generator Files ........................ 412

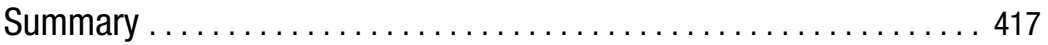

CHAPTER 19 Converting Game Records .................... 419

Converting Our Database to Migrations $\ldots \ldots \ldots \ldots \ldots \ldots \ldots \ldots . \ldots 419$

The Games Table .................................... 421

Cleaning Up the Games Table. ....................... 423

Creating the Games Model ........................... 426

Creating the Developer and Publisher Models. . . . . . . . . . . . . . . 428

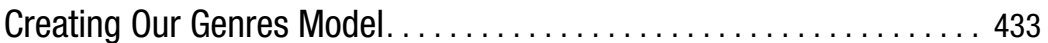

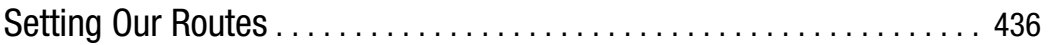

Creating Our Controllers ............................ 436

Creating Our Views .................................. 440

A Standard Layout. ............................... 440

Enhancing Our Layout with Ext Border Layout. . . . . . . . . . . 442

Defining the Workflow .............................. 445

Listing the Current Resource...................... 446

Creating a New Resource ....................... 446

Deleting a Specific Resource ........................ 446

Editing a Specific Resource.......................... 447

Building the Developer Pages. ......................... 447

Listing Our Developers . . . . . . . . . . . . . . . . . . . . . . . . . . . 447

Editing a Developer............................. 452

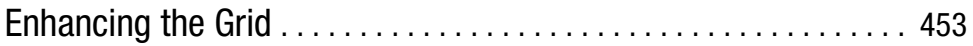

Adding Buttons to Our Toolbar ....................... 462

Deleting a Developer ............................ 463

Creating a New Developer......................... 466

Abstracting Our Workflow into a Helper ................... 468

Building the Show / Edit Template. . . . . . . . . . . . . . . . . . . . . . . . 479

Capturing Failed Creations .......................... 480 
Games.................................... 481

Handling Select Boxes ........................ 486

Providing WYSIWYG Functionality. . . . . . . . . . . . . . . 489

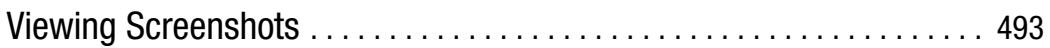

The Screenshot Model ............................... 493

Summary ...................................... 499

CHAPTER 20 Supporting News ............................. 501

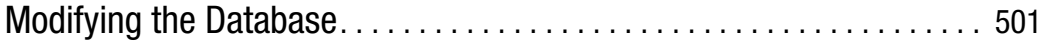

Creating a Model for News .............................. 504

Creating Our Controller ............................. 506

Creating Our Resource .......................... 507

Building Our List View ............................. 507

Redefining the Index Method . . . . . . . . . . . . . . . . 507

A First Pass at the Index Page ...................... 508

Modifying the Grid. ............................ 510

Capturing Creation Errors $\ldots \ldots \ldots \ldots \ldots \ldots \ldots \ldots \ldots \ldots \ldots \ldots 22$

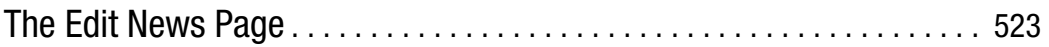

Editing the News Post. .......................... 523

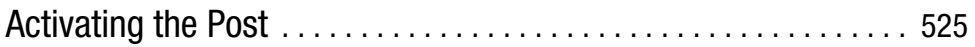

Building Associations to the Post ................... 527

Summary ........................................... 534

CHAPTER 21 Enhancing the Gaming Site $\ldots \ldots \ldots \ldots \ldots \ldots \ldots \ldots$

Build Your Own Generator ............................. 535

Add Login Capabilities ................................ 535

Associate Publishers, Developers, and Consoles to News Posts . . . . . 537

Create a Consoles Constant ................................. 537

Add Box Art and Screenshot Uploads . . . . . . . . . . . . . . . . 537

Add Support for Games Reviews $\ldots \ldots \ldots \ldots \ldots \ldots \ldots \ldots \ldots \ldots$

Add Long Content Support .............................. 538

Add the Acts as Paranoid Plug-In . . . . . . . . . . . . . . . . . . 538

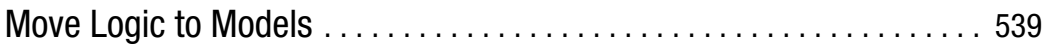

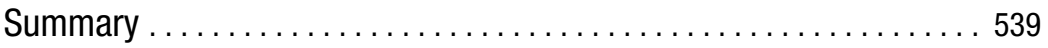




\section{PART 8 Integrating with a RESTful Application Using Edge Rails (Rails 2.0)}

CHAPTER 22 Brief Overview of Highrise 543

Creating a Highrise Account .......................... 544

Creating Contacts ................................... 545

Creating Tasks .................................. 547

Highrise Has More to Offer ........................ 547

A Special Note About Permissions .................... 548

Summary ..................................... 548

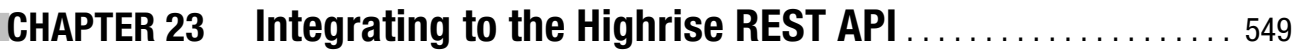

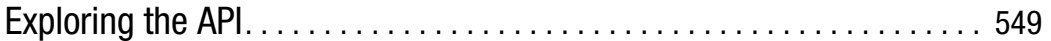

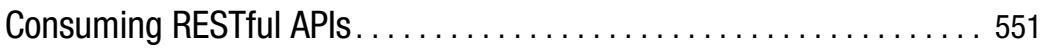

Creating a New Edge Rails Project ..................... 552

Pulling Down the Edge Version of Rails................ 553

Creating an Edge Rails Application ................... 554

Testing Our Connectivity . . . . . . . . . . . . . . . . . . . . . 555

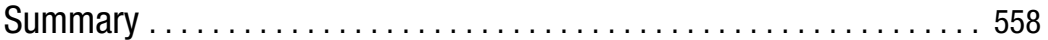

CHAPTER 24 Building the Appointment Scheduler ............... 559

What Are We Going to Build? ............................ 559

Putting Together Our Layout. ........................ 560

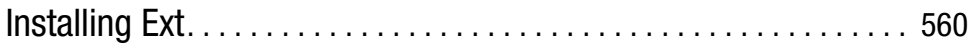

Using Yahoo Maps. ........................... 561

Obtaining an Application ID . . . . . . . . . . . . . . . . . . . . 561

Our Layout Script .............................. 561

The Home Controller .................................. 562

Plugging In Our Map ............................ 565

Adding Our Task List ................................ 566

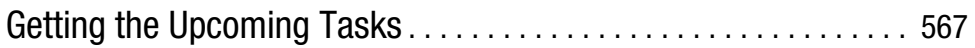

Displaying Our Upcoming Tasks ........................ 569

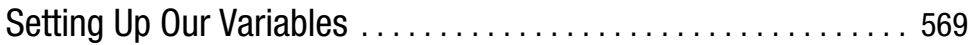

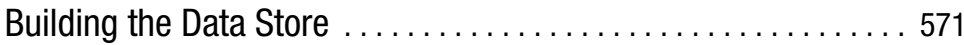

Displaying Customers on the Map ................... 576 
Managing Notes $\ldots \ldots \ldots \ldots \ldots \ldots \ldots \ldots \ldots \ldots \ldots \ldots \ldots \ldots$

Displaying Notes ............................... 579

Adding Notes ............................... 579

Using AJAX to Update Displayed Notes . . . . . . . . . . . 581

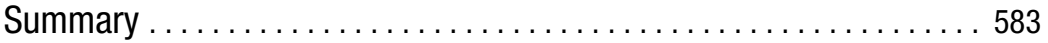

CHAPTER 25 Enhancing Our Rails 2.0 Application $\ldots \ldots \ldots \ldots \ldots \ldots 5$

Enhancing the Highrise Project $\ldots \ldots \ldots \ldots \ldots \ldots \ldots \ldots \ldots \ldots \ldots$

Cache Customer Data. ............................ 585

Create Appointments ............................. 585

Edit a Customer............................... 586

New Features in Edge Rails . . . . . . . . . . . . . . . . . . . 586

Sexy Migrations............................... 586

Automatically Generated Migrations . . . . . . . . . . . . . 587

Database Commands ............................... 587

View Routes .................................. 587

Query Caching $\ldots \ldots \ldots \ldots \ldots \ldots \ldots \ldots \ldots \ldots \ldots \ldots \ldots$

RESTful Routing Improvements .................. 588

Features Removed from the Framework. ............... 588

Summary .................................. 589

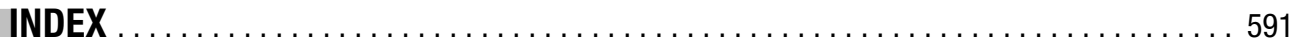




\section{About the Author}

ELDON ALAMEDA is a web developer who currently resides in the harsh climates of Kansas. He develops Ruby on Rails applications for a small technology startup in downtown Kansas City; prior to this, he did development for a variety of companies including local advertising firms, Sprint PCS, and IBM. During the '90s, he also acquired a nice stack of worthless stock options working for dot-com companies. When he's not sitting in front of a computer or irritating his wife by describing a new technology as "sexy," Eldon spends most of his time at home playing games with his young daughter. 


\section{About the Technical Reviewer}

PAUL BENTLEY has been writing software professionally for over a decade. He has experience in many areas of computing, from embedded devices to 3-D graphics. He is especially proficient in the telephony world, experienced with both traditional computer telephony and SIP-based solutions. He is currently working with Rails, developing web applications for corporations who want stable solutions to a variety of problems.

As an avid Go player, he tries to play every day-though he admits he still has a lot to learn before he can even be considered an amateur. He lives with his girlfriend and daughter in Harrogate, UK. If you feel like challenging Paul to a game of Go, he can be tracked down via paulbentley.net. 


\section{Acknowledgments}

B

ah, blah, writing a book is hard, blah, blah.

That being said, this book could not be possible without the help, patience, and wisdom of a number of people.

Keir Thomas, Chris Mills, and Ben Renow-Clarke, the holy trinity of editors who have been involved with this project. Thank you for your guidance, advice, and assistance throughout the project.

Paul Bentley, the technical reviewer. Thank you for the long hours that you spent going through the code in the book, your insightful comments (even when we didn't agree), and your many words of encouragement that helped along the way.

Tracy Brown Collins, the project manager for this book. Thank you for your tireless work at keeping things (which typically meant me) on track. Thank you for also being flexible when times called for it.

Heather Lang, the copy editor. Thanks for all of your suggestions on the text and for the wonderful polish that you applied to the text of this book. You truly went above and beyond for me on numerous occasions, and I want to let you know how much I appreciated it.

Laura Cheu and her production team. Thanks for all of your hard work to help get the book out into the stores as fast as possible and for putting up with my requests for last, last minute changes. You came to my rescue on a number of occasions, and words cannot express my appreciation.

Thanks go to my wife Dori for your kindness, support, encouragement, and for essentially taking on the role of a single mother to support me over the last nine months. And for (almost) never complaining when I asked you to go pick me up an order of hot wings for dinner.

Finally, I'd like to thank my daughter Kaylee for being my sunshine and always bringing a smile to my face. 


\section{Introduction}

P

ractical Rails Projects is for developers who have already read a beginning Rails book (or worked though a series of introductory tutorials online) and are now looking to expand that knowledge by gaining practical experience developing a variety of web applications in Rails. It's for developers who want to gain hands-on experience of building Rails applications that do interesting things such as caching, RESTful routing, using Active Resource and RJS, and connecting Rails to legacy databases. While I do make the assumption that readers have read some previous Rails material, I believe that I've also provided enough review information that a highly motivated reader with previous experience in a model-view-controller framework and familiarity with another object-oriented language should be able to work through the material.

\section{The Problem with Most Training Books and Courses}

Over the course of my career, I've had to sit through an inordinate amount of technical training sessions, and I would estimate that 98 percent of them all suffered from the same major flawthey didn't actually teach anything. I sat in a classroom for a week and proved that I could follow step-by-step directions. Heck, for the majority of them, I could even daydream about other things all day and still pass the course. It wasn't until I got back to the office and was confronted with having to work with the technology myself (without a safety net) that I actually learned anything. It was only when I was removed from the ideal environment and had to use the technology in the real world that its benefits or weaknesses were revealed. I'm sure many of you have suffered through similar things.

Unfortunately, it seems that many current programming books fall into that same trap. The ones that provide instruction on how to build applications tend to fall into this same paintby-numbers mentality so that at the end of the book, you've simply proven that you can follow step-by-step directions as well. I wanted to write something different.

You see, I love Ruby on Rails. I truly believe that it's the best web development framework available today. I know that Rails has brought a lot of the fun of web development back to my work and that it has made me a better developer as well. So when the opportunity to write this book came to me, I wanted to write something that would help others develop the same love for Rails. As I reviewed the existing books available on Rails, I noticed that they fell into just a few categories:

- Introductory Rails books provide the necessary introduction to the structure, conventions, and features of Rails. Examples include Beginning Rails: From Novice to Professional (Jeffrey Allan Hardy, Cloves Carneiro Jr., and Hampton Catlin. Apress, 2007), Agile Web Development with Rails (Dave Thomas, David Hansson, Leon Breedt, and Mike Clark. Pragmatic Bookshelf, 2006), or even Ruby on Rails for Dummies (Barry Burd. For Dummies, 2007). 
- Single-project books are designed to spoon-feed the reader, moving step-by-step through the creation of a single application in Rails, such as building a social network site or an e-commerce site with Ruby on Rails.

- Recipe books are designed for intermediate to advanced Rails developers that include short snippets of code to demonstrate solutions for solving common problems such as adding authentication.

- Reference books are designed for intermediate to advanced Rails developers and take you deeper into a single feature or component related to Rails development, such as the excellent Ruby for Rails: Ruby Techniques for Rails Developers (David Black. Manning Publications, 2006), and books such as Pro ActiveRecord: Databases with Ruby and Rails (Kevin Marshall, Chad Pytel, and Jon Yurek. Apress, 2007), Deploying Rails Applications A Step-by-Step Guide (Ezra Zygmuntowicz and Bruce Tat. Pragmatic Programmer, 2007), and so on.

Typically, I recommend people interested in learning Rails to use books in exactly this order. Start with a beginning Rails book, then move into doing a variety of the project-based books to gain hands-on experience. Finally, move onto the recipe and reference books as a means of deepening your knowledge.

\section{How This Book Is Different}

Of those listed above, I believe that people gain the most knowledge of Rails from the projectbased books. Unfortunately, even when dealing with a subject as fun as Rails, it's still possible to shut off your mind while reading those books and just end up following the instructions without learning from them. That's not meant as a knock on any of those books. I've bought and read pretty much all of them myself.

I wanted to give you something that was focused on helping you bridge that gap from being a beginning Rails developer to becoming an experienced Rails developer. I wanted to give you more value for your book-buying dollar by not just showing you how I might build an application but instead giving you the tools and knowledge necessary to build these applications for yourself.

So, rather than simply taking you through the process of how to build a single solution, we'll tackle several different types of projects. Each project was selected to allow you to develop hands-on experience either working with a core feature of Rails (such as caching or RESTful routing) or to wrangle an interesting problem domain in an effort to spark your interest. Each of these projects could have easily been extended out into the single-project book format.

\section{Learning to Ride a Bike}

In addition, rather than expand each project out into a full book of its own and spoon-feeding you the step-by-step instructions for how to build the exact project that I would build, I chose to scale back the applications a bit, giving you the results of an initial rollout version of each application. This way, each application is workable yet still at a point where you can easily modify and extend it to suit your own needs. When describing this book, I've often used the analogy of teaching someone to ride a bike. My goal is to help you get past that initial hurdle of 
getting up and going — and then let go once you've got some momentum and balance so that you can finish the ride yourself.

At the end of each project, I include a number of exercise ideas for you. As I believe that you often learn some concepts the best when having to fix something broken, I've created some areas in the projects that are less than optimal and then point them out in the exercise sections for you to fix. Other ideas I give you point you in new directions to take our project that would be fun and interesting. I strongly recommend that you go through all of the exercises, as I truly believe that you will learn more from them than in the other sections of the book (which isn't to imply that I haven't tried to share a lot of cool and interesting web development techniques with you in the rest of the book).

\section{What's Not in This Book}

Of course, writing a book like this also requires a substantial amount of sacrifice of subjects that the geek in me would have liked to cover as well. Unfortunately, if I covered everything that I wanted to, this book would have turned out to be around 2,000 pages (and I would probably still be writing it). That said, some of the core elements that I don't cover in the book but are important for you to understand are mentioned in the following sections.

\section{Database Tuning and Indexing}

Since database tuning and indexing are absent, you'll notice that we're not adding any additional indexes onto the databases in most of the applications. This is fine while our data storage is small and our queries remain fairly simple. However, as our applications grow over time, this lack could cause significant performance issues. Perhaps because I came into web development by way of database administration, this was an important feature that was hard for me to remove from the book, but this subject is complex and deserves more attention than I would have been able to give it in this book. Perhaps I'll write a second book that's focused entirely on this subject.

\section{Testing}

Test-driven development (TDD) has certainly taken a strong foothold within the Rails community, and while I haven't yet become a convert to writing tests first, I'm a firm believer in the value of developing a comprehensive test suite before the application is deployed. I've heard it said that all applications are going to be tested-the question is if you'll write the tests yourself or simply dump the application on your users to test it for you.

\section{Refactoring}

Going hand-in-hand with a solid set of test cases is the need to refactor the code. As each project is at an initial rollout stage, there's always going to be lots of room for refactoring the code to make it cleaner, simpler, or in some cases optimized for performance and scaling. 
In the exercise section of each project, I try to point you in the right direction for some common-sense refactorings and optimizations.

\section{Icons Used in This Book}

As much as I would love to be able to claim responsibility for all of the artwork used in this book, the simple truth is that I'm a coder, not an artist. So to give credit where credit is due, here is a short list of the people responsible.

\section{MonkeyTasks Project}

Some icons used in this project were modified versions of ones I downloaded from the Creative Commons Licensed Mini-Icons v2 by Timothy Groves. You can obtain the whole set at http:// www. brandspankingnew. net/archive/2006/12/hohoho.html.

\section{Exercisr Project}

Icons used in this project were from Paul Armstrong's Gallery 2 Icon set, which is licensed under the GNU General Public License (GPL). They are available for download at http: // paularmstrongdesigns.com/portfolio/.

\section{Typo Blog Project}

Icons used in this project were created by Amanda Dinkel, a talented Kansas City graphic designer whose portfolio can be found at http://creativehotlist.com/ index. asp?linkTarget=fullProfile. asp\&indID=83102.

\section{GamingTrend Project}

Icons used in this project were created by Mike Dunn of http://www. foolishstudios. com. For the navigation links, he utilized and modified the Creative Commons licensed FamFam Silk Icons available at http://www. famfamfam.com/lab/icons/silk/. 